$01 ; 14$

\title{
Методика пространственно-временного анализа электрической активности головного мозга
}

\author{
(C) А.Е. Руннова ${ }^{1,2}$, М.О. Журавлев ${ }^{2, \uparrow}$, А.Р. Киселёв ${ }^{1,2}$, А.О. Сельский ${ }^{2}$ \\ ${ }^{1}$ Саратовский государственный медицинский университет им. В.И. Разумовского, Саратов, Россия \\ ${ }^{2}$ Саратовский национальный исследовательский государственный университет им. Н.Г. Чернышевского, Саратов, Россия \\ 『 E-mail: zhuravlevmo@gmail.com
}

Поступило в Редакцию 2 сентября 2019г.

В окончательной редакции 13 марта 2020 г.

Принято к публикации 13 марта 2020г.

\begin{abstract}
Предложен новый метод анализа пространственно-временной динамики паттернов активности головного мозга на базе непрерывного вейвлетного преобразования. Предложенная методика прошла апробацию на примере электрической активности головного мозга, соответствующей планированию двигательной активности. Показано, что данный метод обладает возможностью наглядной детекции возникновения и пространственной динамики частотных паттернов.
\end{abstract}

Ключевые слова: частотно-временной анализ, непрерывное вейвлетное преобразование, обработка биомедицинских сигналов, ЭЭГ-данные.

DOI: 10.21883/PJTF.2020.11.49498.18023

Изучение активности головного мозга в настоящее время привлекает большое внимание исследователей из различных областей науки [1-3]. Одним из направлений исследований в данной области является изучение и выявление характерных особенностей активности с помощью поверхностной электроэнцефалографии (ЭЭГ) головного мозга. Активные исследования в этом направлении связаны с задачами реабилитации нарушения двигательных функций (см., например, работы [4-7] и ссылки в них). Однако до сих пор многие проблемы в этом направлении не решены окончательно, например распознавание начала или конкретного вида реальной и воображаемой двигательной активности [8], что не в последнюю очередь связано с недостатками современных методов оценки сложных сигналов.

B настоящей работе предложена новая методика пространственно-временного анализа динамики паттернов активности головного мозга на базе непрерывного вейвлетного преобразования (НВП) с возможностью наглядных количественных оценок и дальнейшего применения в реальном времени при реализации нейроинтерфейсных устройств. НВП является мощным инструментом анализа нестационарных сложных сигналов и позволяет проводить их одновременное исследование в частотной и временно́й областях $[9,10]$.

Запишем НВП в классическом виде [9]:

$$
W\left(s, t_{0}\right)=\frac{1}{\sqrt{s}} \int_{-\infty}^{+\infty} x(t) \psi^{*}\left(\frac{t-t_{0}}{s}\right) d t,
$$

где $x(t)$ - временна́я реализация анализируемого сигнала, $\psi_{s, t 0}(t)$ - материнский вейвлет, $s-$ временной масштаб, определяющий ширину вейвлета, звездочка комплексное сопряжение. В качестве материнского вей- влета в формуле (1) выбран комплексный вейвлет Морле, часто применяемый при обработке биометрических данных [10]:

$$
\psi(\eta)=(1 / \sqrt[4]{\pi}) \exp \left(j \Omega_{0} \eta\right) \exp \left(-\eta^{2} / 2\right)
$$

с параметром $\Omega_{0}=2 \pi$, обеспечивающим однозначную взаимосвязь между временны́м масштабом $s$ вейвлетпреобразования и частотой $f$ фурье-преобразования, а именно $f=1 / s$ [11]. Далее для удобства будем использовать более привычное понятие частоты $f$. Введем мгновенное распределение энергии НВП

$$
E\left(f, t_{0}\right)=\left|W\left(f, t_{0}\right)\right|^{2} .
$$

Воспользуемся упрощением частотно-временного представления сигналов на основе скелетонов $s c$ НВП [12,13]. При использовании скелетонов $s c$ для каждого момента времени $t_{0}$ рассматриваются только частоты $f_{m}$, на которые приходятся максимальные значения $\max \left(E\left(f_{m}, t_{0}\right)\right)$ мгновенной энергии (3), т. е. $E\left(f_{m}, t_{0}\right)>E\left(f_{k}, t_{0}\right)$ для $\forall f_{k} \in f_{m} \pm \Delta$. Для времени $t_{0}$ можно ввести неограниченное количество скелетонов $s c^{i}$, старший из которых $s c^{1}$ будет отвечать глобальному максимуму энергии (3), а остальные локальным максимумам в порядке убывания. Для анализа ЭЭГ достаточно оценки трех скелетонов. В то же время, хотя скелетонное представление эффективно для одномерного анализа, при многоканальном исследовании возрастающий объем данных затрудняет анализ и визуальное представление результатов. Для решения этого предлагается следующая методика.

Разобьем весь частотный диапазон на шесть интервалов: $\delta(1-4 \mathrm{~Hz}), \theta(4-8 \mathrm{~Hz}), \alpha(8-12 \mathrm{~Hz}), \beta_{1}(12-20 \mathrm{~Hz})$, $\beta_{2}(20-30 \mathrm{~Hz})$ и $\gamma(30-40 \mathrm{~Hz})$, принятых в нейрофизиологии [14]. Далее для каждого канала ЭЭГ в момент 

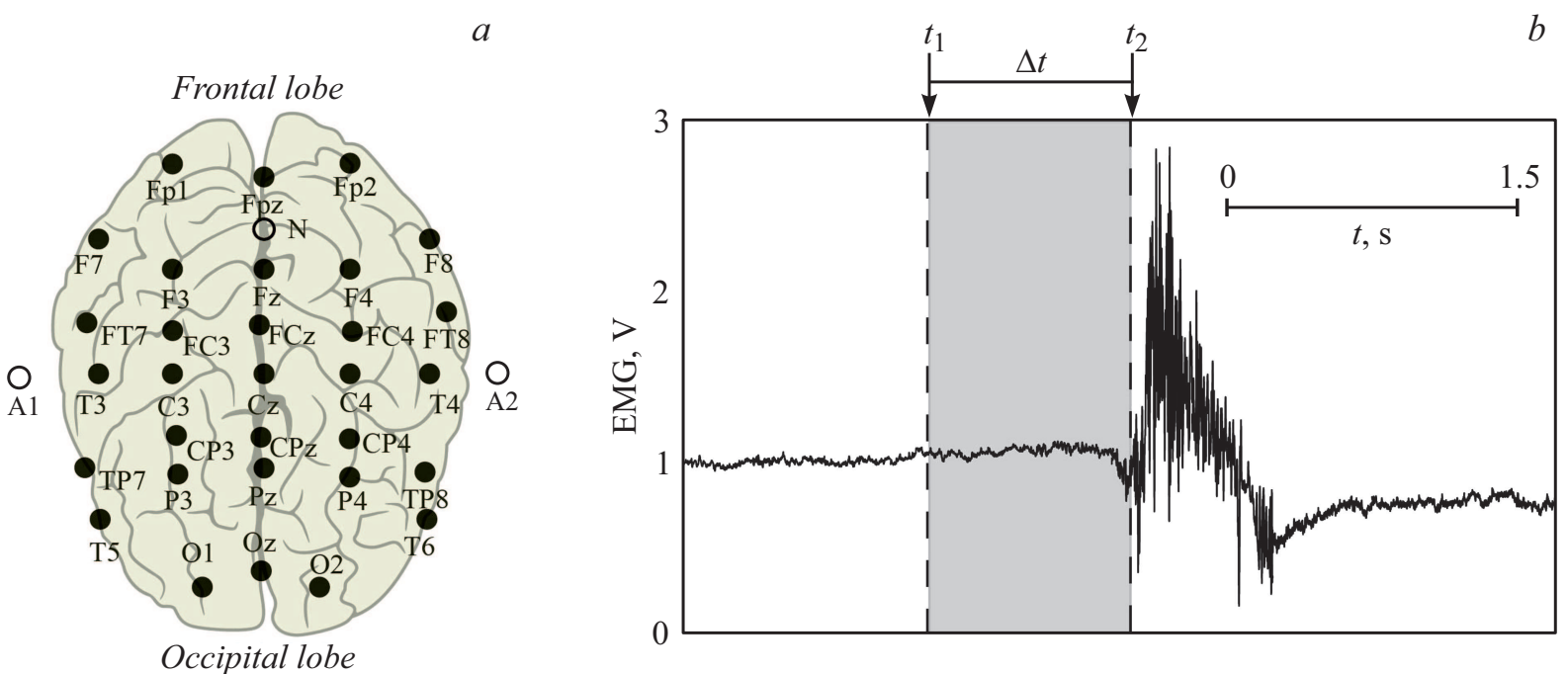

Рис. 1. $a-$ схема „10-20“ расположения именованных электродных отведений ЭЭГ, $b-$ график электромиографического сигнала, регистрируемого в правом предплечье у испытуемого.


Рис. 2. Различные сценарии $(a-c)$ возможных пространственных распределений по скальпу паттернов $\left\langle A_{i}^{j}\right\rangle$ ЭЭГ-активности на четырех стадиях $\Delta t^{1,2,3,4}$ периода подготовки движения.

времени $t_{0}$ введем шесть характеристик, определяющих искомый сигнал в указанных интервалах частот:

$$
E_{i}^{\delta, \theta, \alpha, \beta_{1}, \beta_{2}, \gamma}(t)=\sum\left\{\begin{array}{l}
1 \text { при } f_{\min }^{\delta, \theta, \alpha, \beta_{1}, \beta_{2}, \gamma}<s c_{i}^{1}<f_{\max }^{\delta, \theta, \alpha, \beta_{1}, \beta_{2}, \gamma} ; 0 \text { в других случаях, } \\
0.5 \text { при } f_{\min }^{\delta, \theta, \alpha, \beta_{1}, \beta_{2}, \gamma}<s c_{i}^{2}<f_{\max }^{\delta, \theta, \alpha, \beta_{1}, \beta_{2}, \gamma} ; 0 \text { в других случаях, } \\
0.25 \text { при } f_{\min }^{\delta, \theta, \alpha, \beta_{1}, \beta_{2}, \gamma}<s c_{i}^{3}<f_{\max }^{\delta, \theta, \alpha, \beta_{1}, \beta_{2}, \gamma} ; 0 \text { в других случаях, }
\end{array}\right.
$$

где $i$ - номер канала ЭЭГ, $s c_{i}^{1,2,3}-$ частота первого, второго и третьего скелетона для $i$-го канала. Теперь введем 
оценку интенсивности $A_{i}^{j}$ для частотного диапазона $j$ в $i$-м канале в момент времени $t_{0}$ следующим образом:

$A_{i}^{j}\left(t_{0}\right)=\frac{E_{i}^{j}\left(t_{0}\right)}{E_{i}^{\delta}\left(t_{0}\right)+E_{i}^{\theta}\left(t_{0}\right)+E_{i}^{\alpha}\left(t_{0}\right)+E_{i}^{\beta_{1}}\left(t_{0}\right)+E_{i}^{\beta_{2}}\left(t_{0}\right)+E_{i}^{\gamma}\left(t_{0}\right)}$,

где $j$ - один из частотных диапазонов $\left(\delta, \theta, \alpha, \beta_{1}, \beta_{2}, \gamma\right)$. Если $A_{i}^{j}>0.8$, то динамика данного частотного диапазона $j$ доминирует над остальными в рассматриваемый момент времени $t_{0}$.

В таком случае для каждого $t_{0}$ можно построить пространственное распределение доминирующих частотных диапазонов, что позволяет эффективно производить ЭЭГ анализ и визуализировать получаемые результаты.

Разработанная методика пространственно-временного детектирования частотных паттернов была апробирована на реальных данных двигательной активности. В экспериментальных работах приняли участие 15 здоровых испытуемых, у каждого из которых регистрировались $n=31$ каналов ЭЭГ на базе электроэнцефалографа „Энцефалан-ЭЭГР-19/26“ монополярным способом на основе схемы „10-20“[15], представленной на рис. 1, $a$. Для контроля момента начала движения человека использовалась регистрация электромиографического сигнала с предплечий рук испытуемого. В эксперименте испытуемый по подаваемым звуковым сигналам сжимал/разжимал кулак левой/правой руки. Длительности пауз между звуковыми командами задавались случайным образом с достаточно широким разбросом (более $4 \mathrm{~s}$ ) для исключения возможности привыкания.

Анализ ЭЭГ проводился на этапе подготовки двигательной активности, который строго определялся как временной интервал от подачи звуковой команды до момента начала движения, определяемого по электромиографическому сигналу (рис. 1,b). Далее весь временной интервал $\left[t_{1} ; t_{2}\right]$ был разделен на четыре равные стадии, временна́я длительность которых определялась как $s=0.25\left(t_{2}-t_{1}\right)$. Обозначим временны́е стадии в процессе подготовки двигательного акта $\Delta t^{1}\left[t_{1} ; t_{1}+s\right], \Delta t^{2}\left[t_{1}+s ; t_{1}+2 s\right], \Delta t^{3}\left[t_{1}+2 s ; t_{1}+3 s\right]$, $\Delta t^{4}\left[t_{1}+3 s ; t_{2}\right]$. Для каждой стадии были рассчитаны усредненные характеристики интенсивности $A_{i}^{j}(5)$ :

$$
\left\langle A_{i}^{j}\right\rangle_{\Delta t}=\frac{\int_{\Delta t} A_{i}^{j}(t) d t}{\Delta t} .
$$

На рис. 2 представлены результаты выполненного расчета $\left\langle A_{i}^{j}\right\rangle$ (6) по стадиям $\Delta t^{1,2,3,4}$, демонстрирующие три характерных сценария пространственно-временной динамики $\alpha$ - и $\beta_{2}$-активности (части $a, b$ и $c$ соответственно). Отметим, что общей закономерностью наблюдаемой динамики является смещение $\alpha$-паттерна к затылочной зоне и развитие $\beta$-паттерна в теменной и лобной долях, при этом в сценарии 1 этот процесс занимает меньше времени, чем в сценарии 2. Сценарий 3 демонстрирует большую площадь $\alpha$-паттерна на стадиях
$\Delta t^{1,2}$ и $\beta$-паттерна на стадиях $\Delta t^{3,4}$, этот сценарий наблюдался у $73.3 \%$ группы (11 испытуемых).

Полученные результаты анализа пространственновременной картины эволюции электрической активности головного мозга, соответствующие планированию двигательной активности, хорошо соотносятся с данными ранее известных работ [5-7], что доказывает корректность предложенной методики. Кроме того, поскольку в основе методики лежит НВП, ее реализация возможна на базе технологии CUDA в реальном времени с использованием подходов, изложенных в работе [16], что открывает перспективы применения данной методики в разработке нейроинтерфейсов, например, для задач нейрореабилитации больных с нарушением двигательных функций.

\section{Финансирование работы}

Работа в части разработки методов анализа данных выполнена при финансовой поддержке Совета по грантам Президента РФ для государственной поддержки молодых российских ученых (проект МК-1599.2019.9). Экспериментальные процедуры по регистрации и анализу физиологической активности проведены в рамках проекта Российского фонда фундаментальных исследований (№ 20-02-00752 А).

\section{Соблюдение этических стандартов}

Все процедуры, выполненные в исследовании с участием людей, соответствуют этическим стандартам институционального и/или национального комитета по исследовательской этике и Хельсинкской декларации 1964 г. и ее последующим изменениям или сопоставимым нормам этики.

От каждого из включенных в исследование участников было получено информированное добровольное согласие.

\section{Конфликт интересов}

Авторы заявляют, что у них нет конфликта интересов.

\section{Список литературы}

[1] Рабинович М.И., Мюезинолу М.К. // УФН. 2010. Т. 180. № 4. C. 371-378.

[2] Meng J., Edelman B.J., Olsoe J., Jacobs G., Zhang S., Beyko A., He B. // Front. Neurosci. 2018. V. 12. P. 227.

[3] Erickson M.A., Kappenman E.S., Luck S.J. // Biol. Psychiatry: Cogn. Neurosci. Neuroimaging. 2018. V. 3. N 1. P. 4-6.

[4] Karimi F., Kofman J., Mrachacz-Kersting N., Farina D., Jiang N. // Front. Neurosci. 2017. V. 11. P. 356.

[5] Neuper C., Scherer R., Reiner M., Pfurtscheller G. // Cogn. Brain Res. 2005. V. 25. N 3. P. 668-677.

[6] Marquez-Chin C. // Signal and Information Processing (GlobalSIP). Global Conf. on IEEE. 2017. P. 428-431.

[7] Hampstead B.M., Towler S., Stringer A.Y., Sathian K. // Alzheimer's \& Dementia: Diagnosis, Assessment \& Disease Monitoring. 2018. V. 10. P. 76-85. 
[8] Blankertz B., Dornhege G., Krauledat M., Mïler K.-R., Curio G. // NeuroImage. 2007. V. 37. N 2. P. 539-550.

[9] Короновский А.А., Храмов А.Е. Непрерывный вейвлетный анализ и его приложения. М.: Физматлит, 2003. 176 с.

[10] Hramov A.E., Koronovskii A.A., Makarov V.A., Pavlov A.N., Sitnikova E.Y. Wavelets in neuroscience. Heidelberg-N.Y.-Dordrecht-London: Springer, 2015. 318 p.

[11] Grubov V., Runnova A., Zhuravlev M., Maksimenko V., Pchelintseva S., Pisarchik A. // Cybernetics and Physics. 2017. V. 6. N 3. P. $108-113$.

[12] Runnova A.E., Zhuravlev M.O., Pisarchik A.N., Khramova M.V., Grubov V.V. // Proc. SPIE. 2017. V. 10063. P. 1006319.

[13] Keirn Z.A., Aunon J.I. // IEEE Trans. Biomed. Eng. 1990. V. 37. N 12. P. $1209-1214$.

[14] Niedermeyer E. Niedermeyer's electroencephalography: basic principles, clinical applications, and related fields. Lippincott Williams \& Wilkins, 2011. 1296 p.

[15] Руннова А.Е., Максименко В.А., Пчелинцева С.В., Куланин Р.А., Храмов А.Е. // Информационно-управляющие системы. 2018. № 1. С. 106-115.

[16] Грубов В.В., Недайвозов В.О. // Письма в ЖТФ. 2018. T. 44. B. 10. C. 103-110. 